\title{
ARTÍCULOS
}

\section{ROY WAGNER (1938-2018): EL ESPEJO HUMEANTE DE LA ANTROPOLOGÍA*}

\author{
ROY WAGNER (1938-2018): THE SMOKING MIRROR OF ANTHROPOLOGY
}

\author{
Olatz G. Abrisketa ${ }^{1}$
}

Universidad del País Vasco (UPV/EHU)

Recibido: 1 de abril de 2019; Aprobado: 14 de junio de 2019; Publicado online: 27 de noviembre de 2019

Cómo citar este artículo / Citation: Abrisketa, Olatz G. 2019. «Roy Wagner (1938-2018): el espejo humeante de la antropología». Disparidades. Revista de Antropología 74(2): e016. doi: <https://doi.org/10.3989/dra.2019.02.016>.

RESUMEN: Roy Wagner murió en septiembre de 2018. Este artículo, escrito desde el afecto y la admiración, muestra la originalidad del pensamiento de Wagner y destaca la importancia del mismo para entender no solo la antropología actual, cada vez más influenciada por su trabajo, sino también el propio proceder antropológico y su gran invención: la cultura. El artículo se acerca a momentos de su biografía, a sus gustos y relata anécdotas por él contadas para entender mejor los vínculos disciplinares y teóricos que Wagner establece con autores como Claude Lévi-Strauss, Victor Turner, Gregory Bateson o Carlos Castaneda. El artículo incluye al final una amplia bibliografía suya para quienes quieran profundizar en la obra de un autor que va a marcar sin duda la antropología de los próximos tiempos.

PALABRAS CLAVE: Roy Wagner; Invención; Cultura; Obviación; Giro ontológico.

ABSTRACT: Roy Wagner died in September 2018. This article, written out of affection and admiration, shows the originality of Wagner's thought and highlights its importance for understanding, not only current anthropology, increasingly influenced by his work, but also the anthropological machine and its great invention, culture. The article examines moments of his life and his tastes, and recounts anecdotes told by him to provide a better understanding of the disciplinary and theoretical links that Wagner established with authors like Claude Lévi-Strauss, Victor Turner, Gregory Bateson and Carlos Castaneda. The article concludes with an extensive bibliography for those who want to explore further the work of an author who is set to become a defining figure in the anthropology of the near future.

KEYWORDS: Roy Wagner; Invention; Culture; Obviation; Ontological Turn.

Copyright: (C) 2019 CSIC. Este es un artículo de acceso abierto distribuido bajo los términos de la licencia de uso y distribución Creative Commons Reconocimiento 4.0 Internacional (CC BY 4.0).

* Conocí personalmente a Roy Wagner y pude profundizar en su trabajo gracias a una ayuda de movilidad (UPV/EHU MOVGV16/04) otorgada por el Gobierno vasco para realizar una estancia de investigación en el Departamento de Antropología de la Universidad de Virginia entre enero y junio de 2016. Roberto Armengol, Emily Cone-Miller y Sheena Singh hicieron aquella estancia más acogedora y divertida y quiero mandarles desde aquí un afectuoso agradecimiento. Quiero agradecer también a Roy su cercanía, generosidad y humor, y a Ida Hoequist el haberme grabado las clases del último curso que aquel ofreció sobre "Don Juan \& Castaneda" en 2018. Por último, indicar que este trabajo forma parte del Grupo de Investigación Consolidado Tipo A del Sistema Universitario Vasco IT1199-19.

1 Correo electrónico: olatz.gonzalez@ehu.eus. ORCID iD: <https://orcid.org/0000-0001-8644-8671>. 

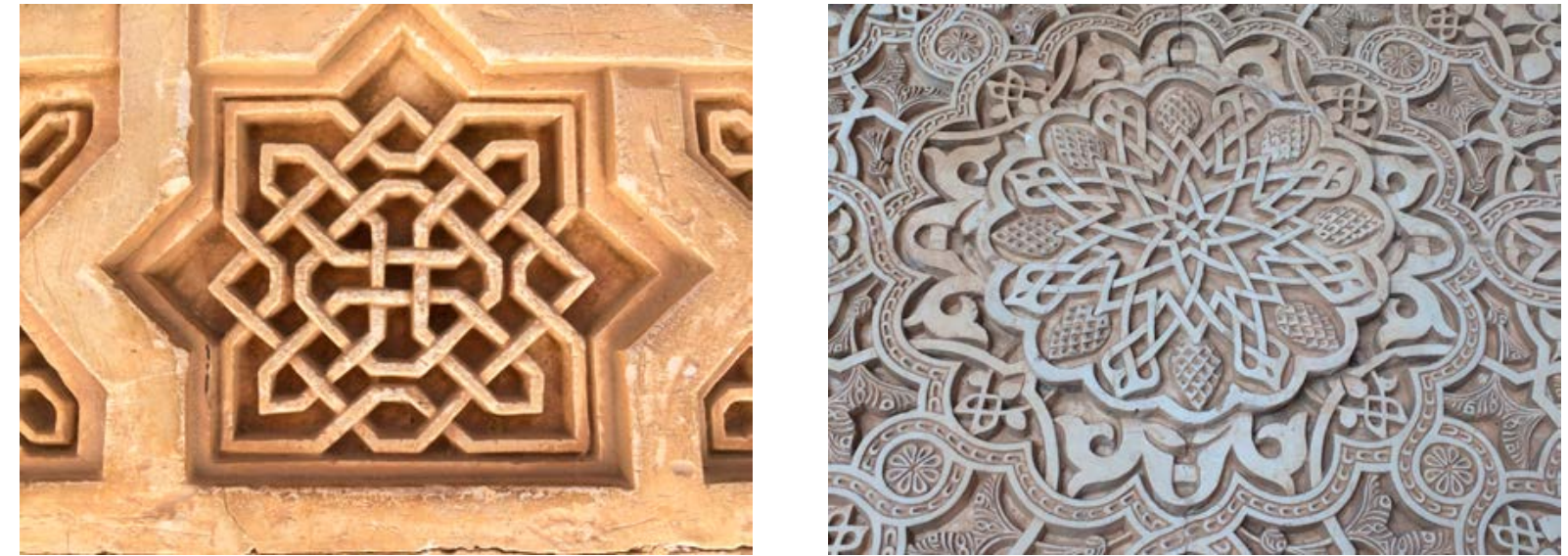

FIgURA 1.-Motivos decorativos de la Alhambra, Granada. Septiembre de 2018.

El pasado 10 de septiembre de 2018 Roy Wagner moría en su casa de Charlottesville, Virginia. Tres días antes de su muerte, aprovechando el congreso de AIBR en Granada, fotografié varios patrones decorativos en la Alhambra para enviárselos (Figura 1). Sabía que esas geometrías de escayola, en las que las líneas se entrelazan generando isometrías y trayectos invertidos, iban a deleitar sobremanera a Roy, quien tenía su despacho repleto de imágenes insólitas como el triángulo de Penrose, el símbolo de Hunab Ku o la diosa Khali bailando con su sombra frente al espejo (Figura 2). Las remití igualmente para acompañar el homenaje que le hicieron sus colegas del departamento de antropo- logía de la Universidad de Virginia (UVa), donde daba clases desde 1974. Al recibirlas, Fred Damon me contestó que podía suponer qué estaba haciendo Roy con aquellas imágenes en ese mismo momento. No añadió nada más, pero pude imaginármelo acompañando con ellas el segundo movimiento de la Heroica, descifrando algún acertijo Barok o simplemente utilizándolas para orientarse por esa tercera dimensión donjuaniana en la que ahora se encontraba. Roy se escoró hacia la antropología porque consideraba que los historiadores (estudió Historia Medieval en Harvard) se limitaban a pensar cronológicamente, careciendo de pensamiento transversal, paradigmático, precisamente el tipo de

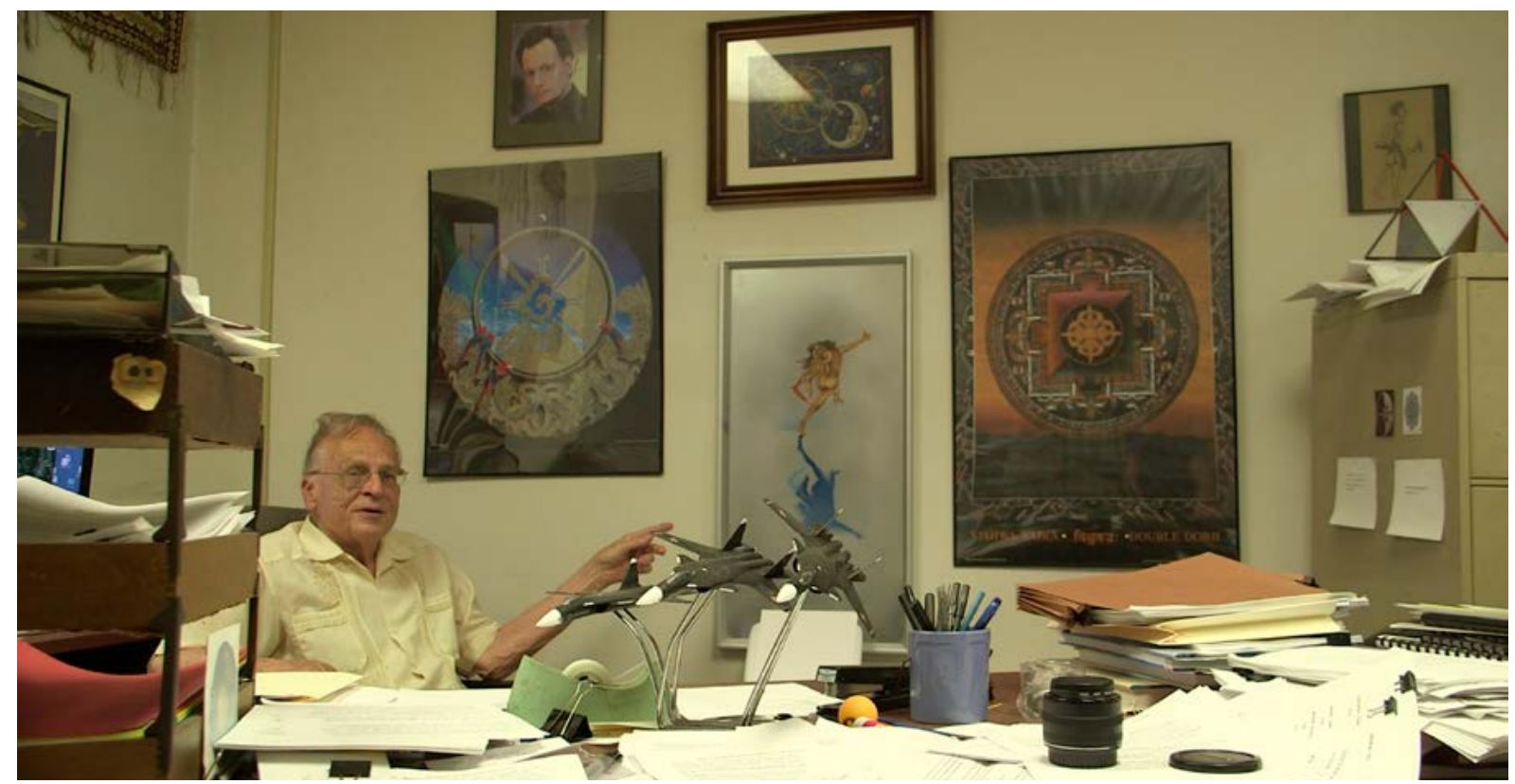

Figura 2. - Roy Wagner en su despacho de Brooks Hall, en la Universidad de Virginia, Charlottesville, en mayo de 2016. 
pensamiento que para él distinguía a la antropología y la convertía en un saber de resonancias (Wagner 2019).

Roy Wagner comprendió como nadie nuestra disciplina, pero lo hizo mucho antes de que fuéramos capaces de entenderlo. En 1975 publicó su tercer y más destacado libro: The invention of culture. Esta obra es seguramente el libro que más radicalmente revela el proceder antropológico y su gran invento: la cultura. En un momento en que la cultura se coronaba como el gran objeto de la antropología (recordemos que el artículo de Clifford Geertz «El impacto del concepto de cultura en el concepto de hombre» se había publicado en 1966 y el volumen La interpretación de las culturas en 1973), un joven Roy Wagner destripa paso a paso en qué consiste esa gran invención que hace posible e inventa a su vez la antropología. En un texto tan denso como iluminador, Wagner presenta el hecho cultural -que la cultura refiera tanto a la especificidad del ser humano como a su diversidad- como el rudimento básico sobre el que se pone a andar la antropología y que marca su carácter y su destino, que no es otro que la relatividad. Esta relatividad no es análoga a la del relativismo cultural (quizás la postmodernidad en antropología nunca se habría planteado del mismo modo si el texto de Wagner hubiera sido ampliamente comprendido en su momento). No es una relatividad consumada, entendida en términos absolutos y que descansa sobre la equivalencia de las culturas. La relatividad a la que apela Wagner es originaria. Las culturas son relativas porque solo se revelan, o más bien se inventan, en relación. Es decir, la cultura no existe hasta ser confrontada y esto siempre se produce en un contexto particular en el que ambas partes deben inventar al otro desde los parámetros de su propio contexto y al hacerlo están inventándose a sí mismos y al propio concepto de cultura:

la cultura en la cual uno crece nunca es realmente "visible» - se da por sentada y sus suposiciones son autoevidentes. Es solo a través de la «invención» de este tipo que el significado abstracto de la cultura (y de muchos otros conceptos) puede entenderse, y solo a través de la experiencia de la diferencia que su propia cultura deviene "visible». En el acto de inventar otra cultura, el antropólogo inventa la suya, y de hecho reinventa la propia noción de la cultura (Wagner 1981: 4).

Esta afirmación, por obvia, quizás nos parezca hoy autoevidente y no en sí misma un descubrimiento de Wagner, sino implícita en todo trabajo antropológico de mérito. Quizás la podamos identificar en Franz Boas, Ruth Benedict, por supuesto Claude LéviStrauss, Clifford Geertz y otra/os. Sin embargo, la gran aportación de Roy Wagner es mostrar cómo esa obviedad ha sido precisamente obviada para que la antropología haya podido ser y seguir siendo inventada: «La antropología es el estudio del ser humano "como si" hubiera cultura» (Wagner 1981: 10). En ese como si radicaría su efectividad, en dar por hecho una realidad que en sí misma es una invención de la propia antropología o sobre la que la propia antropología se ha erigido como disciplina. Este procedimiento de invención no es, sin embargo, exclusivo de la antropología. No solo la antropología procede por medio de la invención de su propio objeto. La invención es algo que se produce objetivamente en el proceso mismo de conocimiento. La gravedad, la selección natural, el neorrealismo, pi, el Superyo, o la naturaleza -el gran invento occidental-, son todas invenciones en el mismo sentido que lo es la cultura. Toda ciencia, saber y conocimiento humano es inventado, algo que Wagner no es el primero ni el único en afirmar, pero que él se ocupa de rastrear a lo largo de su obra.

En 1961, una vez acabado su grado en Historia Medieval, Roy Wagner se traslada a Chicago para realizar el doctorado de antropología bajo la supervisión de David M. Schneider, quien en ese momento está inmerso en el Kinship Project. Aquel proyecto investigaba las consideraciones sobre el parentesco de las familias de clase media americana, y dio lugar a American Kinship (1968), en el que Schneider defenderá que los significados que los americanos atribuyen a las nomenclaturas del parentesco tienen más fuerza a la hora de conformar la familia que los hasta entonces definitorios vínculos de sangre. Con este trabajo se inaugura la dimensión simbólica del parentesco, inscribiéndose este en la corriente culturalista que se está gestando en Estados Unidos en ese momento. Siguiendo la línea de trabajo de su mentor, a finales de 1963, Wagner se va a hacer trabajo de campo a Mount Karimui, en Nueva Guinea. Allí permanecerá durante año y medio estudiando, entre otras cosas, las filiaciones y adscripciones clánicas de los daribis, un grupo de 3000 personas normativamente patrilineales pero cuya adscripción al clan paterno depende del cumplimiento de una serie de pagos a los parientes maternos, principalmente al hermano de la madre. Este tributo se sanciona en relación a la maldición de Souw, héroe mítico que da nombre al libro (The Curse of Souw: principles of Da- 
ribi clan definition and alliance in New Guinea, 1968) y cuyas amenazas de sustracción del alma encuentran un paralelismo con las del hermano materno, quien puede alejar al niño del clan paterno. La necesidad de poner freno al peligro de apropiación del niño por parte del clan de la madre, que se condensa corporalmente en un aumento de la sangre materna frente al semen paterno, obliga al padre a mantener los pagos y son estos los que marcan para Wagner los límites y, por tanto, de-finen los clanes patrilineales. Wagner invierte de este modo el modelo de alianza levi-straussiano para defender que entre los daribis el intercambio de bienes no genera las alianzas sino que define la filiación, mientras que es la consanguinidad la que emparenta: «Exchange defines, consanguinity relates» (El intercambio define, la consanguinidad emparenta).

Desde este primer libro, el trabajo de Wagner dialoga permanentemente con la obra de Claude LéviStrauss (en especial con El pensamiento salvaje). Ambos autores transitan del parentesco a la mitología, encontrando en esta última el territorio ideal para su proyecto teórico; el francés en busca de las estructuras elementales de la mente, el americano de «la completa anatomía de la invención» (Wagner 1981: 157). Ya desde su segundo libro, Habu. The Innovation of Meaning in Daribi Religion (1972), Wagner presenta la que será su mayor preocupación: la producción de sentido; y el presupuesto que dinamizará su obra: toda innovación cultural (luego invención) se genera en un proceso dialéctico entre lo convencional y lo diferenciador-singularizante. El ritual de curación habu, en el que los hombres solteros daribis, habubidi, personifican (impersonate) fantasmas y las mujeres casadas se visten y comportan como hombres, sirve a Wagner para ofrecer una explicación en términos de oposiciones convencionales que son escenificadas y enfrentadas a las potencialidades transformadoras del mundo y de la individualidad. Este recurso a oposiciones binarias y a las relaciones de complementariedad y contraste que se establece entre ellas ha llevado a algunos a considerar el carácter estructuralista de la obra de Wagner (Young 1974; Erikson et al 2008). Sin embargo, a pesar de que su obra está repleta de diagramas que acompañan complejas explicaciones sobre las relaciones entre los elementos en juego, Wagner rechaza la arbitrariedad de la significación sobre la que se funda el estructuralismo y abomina de la reducción de la metáfora a simple función mental o engranaje del significante: la metáfora es «un significante que guarda una rela- ción determinada y no arbitraria con el significado» (Wagner 1972: 169). De ahí su crítica a la decisión de Lévi-Strauss de privilegiar la homología en la explicación del totemismo en detrimento de la analogía (Ludvigson 1974; Wagner 1975; Dulley 2015: 93-102). Además de provocar el distanciamiento entre el modelo o representación y la cosa representada, no reconocer las relaciones analógicas que exhibe el totemismo es para Wagner anteponer los procedimientos implícitos del análisis semiótico a la realidad nativa. Este compromiso con el campo, lugar privilegiado desde el que emana no solo la experiencia dialéctica de la alteridad sino también la energía conceptual de la antropología, ha sido lo que ha colocado a Wagner como gran progenitor del giro ontológico. Un análisis pormenorizado de sus préstamos a esta corriente puede encontrarse en la reciente obra de Martin Holbraad y Morten Axel Pedersen (2017: 69-109) The Ontological Turn, y más someramente en castellano en nuestro artículo "La apertura ontológica de la antropología contemporánea» (González-Abrisketa y Carro-Ripalda 2016).

Siguiendo con la oposición de Wagner a la arbitrariedad del sentido, esta no debe confundirse con la de, por ejemplo, Victor Turner, con quien coincidió en UVa entre 1976 y 1983, año de la muerte de este. Turner, siguiendo en esto a Carl Jung, considera el símbolo la mejor expresión posible de un hecho relativamente desconocido pero que se postula como existente, rechazando la escisión que el planteamiento estructuralista genera entre lo sensible y lo inteligible. Para Wagner (1981: 42), «nuestros símbolos no se relacionan de ningún modo con una "realidad" externa; a lo sumo refieren a otra simbolización que percibimos como realidad». En este sentido, es el reconocimiento de la convención cultural como realidad implícita en toda significación lo que aleja a Wagner del estructuralismo. La oposición conciencia/ exterior (entiéndase en el caso de Wagner exterioridad colectivamente convenida) propia del estructuralismo hace inviable la reversibilidad implícita en todo sentido. Esta reversibilidad posibilita precisamente la invención cultural.

\footnotetext{
Eso implica que el núcleo de la cultura no sería un montaje aleatorio de costumbres, ideas, objetos, instituciones, palabras, y similares, sino un flujo coherente de imágenes y analogías, que no pueden ser comunicadas directamente de mente a mente, sino solamente suscitado, acotado, representado. No está constituido por los símbolos de referencia convencionales, ni por los perceptos privados del
} 
individuo sobre "las cosas en el mundo", sino dentro de una dialéctica reversible que se mueve entre esos límites. Esto hace posible el fenómeno de los tropos suscitados colectivamente así como la incrustación de la referencia convencional dentro de los mundos privados de la percepción individual (Wagner 1986: 129).

El énfasis en el entre, una preposición que implica cierta correspondencia entre los elementos implicados en la relación, pero que sobre todo abre un espacio intersticial siempre enigmático, muestra una pulsión en Wagner en la que puede resonar del mismo modo la obra de Turner. A pesar de que hay pocas referencias cruzadas entre ambos, Victor Turner era el editor general de la serie «Symbol, Myth and Ritual» de la Cornell University Press, en la que Wagner publicó Lethal Speech: Daribi Myth as Symbolic Obviation (1978), el tercer libro de su inintencionada trilogía en torno a la dialéctica entre convención e invención en la actividad creativa del tropo. El primero sería Habu (1972) y el segundo The Invention of Culture (1975). Wagner, por su parte, apela en varios de sus escritos a conceptos como liminalidad y antiestructura para acompañar sus ideas, sobre todo las de obviación y transposición de subjeto y objeto, que referiremos más adelante. A falta de trabajos que estudien las confluencias y diferencias entre ambos autores, hay en ellos cierta preocupación por abordar el punto ciego del estructuralismo. Victor Turner se apoyó para ello en la hermenéutica y propuso el término de "anti-estructura» para hacer inteligible el momento ritual o performativo en que las sociedades, adoptando simultáneamente el papel de sujeto y objeto, se abren a la posibilidad de ser transformadas. La hegemonía de la estructura en la imposición del sentido, sin embargo, no parece tambalearse con la propuesta turneriana, ya que la antiestructura entra en relación de complementariedad con la primera. Roy Wagner, por su parte, con un panteón ecléctico en el que desde el principio moraran el propio Lévi-Strauss y, por supuesto, Gregory Bateson y al que se unirán más tarde Carlos Castaneda y Ludwig Wittgenstein, ofrecerá un concepto que entra en relación innovadora o dialéctica, por utilizar su propio vocabulario, con el de estructura: la obviación, probablemente la propuesta más original y celebrada de Roy Wagner.

La obviación es el procedimiento por medio del cual es posible y se produce toda innovación/invención. Opera por substitución, mejor dicho, por subsunción, en tanto que aquello que es sustituido per- manece obviado en la nueva elaboración o relato. Una relación convencional entre dos términos o realidades es subsumida por una relación innovadora, de ahí que la invención se conciba como un fluir analógico de metáforas: «toda metáfora implica una relación y la innovación se produce por la acumulación de metáforas sobre metáforas, haciendo que las expresiones y significados previos queden subsumidos (por obviación) en las nuevas elaboraciones». Para Wagner, la obviación opera todo el rato y en todos los niveles. Desde un simple lapsus linguae, un chiste o juego de lenguaje hasta manifestaciones culturales y sociales de todo tipo: costumbres, rituales, ciencia, música, moda, ordenamientos jurídicos, arte. Convención e invención se están recreando mutua y constantemente, se están contrainventando por medio de la obviación. Por ello, no debemos concebir la invención como un acontecimiento insólito, sino como algo tan habitual y corriente como la convención. Ambas coexisten en trato de mutua recreación.

Richard Schechner, un íntimo colaborador de Turner en los estudios sobre performance, ofrecerá un concepto que Roy Wagner referirá como análogo al suyo y que puede ayudarnos a abordar la complejidad de la obviación. Es el concepto de no no (not not). Schechner afirma que al actuar, el actor o la actriz tiene una experiencia de sí mismo/a mediada por representar a otros que le hace tener no un yo, sino un no no yo. Volviendo al habu, ritual que solo se celebra ante la amenaza de extinción del grupo por contagio de una mala muerte, cuando los habubidi personifican fantasmas, seres que no están sometidos a la mortalidad humana pero que viven gracias a las personas que poseen, están burlando la mortalidad (o inventando la inmortalidad) al generar una nueva modalidad de no no mortal. Esta operación de desplazamiento metafórico, en la que se inventa una clase original de ser, no afecta solo al significado, sino también al conjunto de relaciones que componen toda cultura, ya que «cualquier innovación significativa introducida en la cultura automáticamente ensancha y participa de aquellas relaciones» (Wagner 1972: 168).

A pesar de que la obviación opera en todos los niveles de la cultura, es en los mitos donde este procedimiento se manifiesta abiertamente. Los mitos en sí mismos no serían sino expresión y celebración del procedimiento de obviación implícito en toda invención cultural. En este sentido, Wagner se alinea con los postulados de Lévi-Strauss, quien también consi- 
deraba que los mitos no apelan a ninguna realidad externa más allá de sí mismos. Los mitos son enunciados autorreferenciales, en los que se ponen en juego las reglas estructurales con las que el inconsciente hace uso del maremágnum de datos almacenados en el subconsciente. En una consideración análoga, aunque diferente en el sentido planteado anteriormente, en el que existe una reversibilidad entre la conciencia y cierta exterioridad convencional a la que la acción del mito muestra el camino de la invención, Wagner considera que los «mitos o los cuentos populares eran instrumentos creativos de propósito (intent), verdaderos hechizos a través de los cuales se producían increíbles transformaciones» (Wagner 2010: 123), es decir, fórmulas de invención para que las sociedades ensayaran sobre sus regulaciones protocolarias (Weiner 1995). Una de las asignaturas que Roy Wagner impartía en UVa, Mythodology, consistía precisamente en analizar este procedimiento. Cada alumna/o elegía el mito o cuento popular que deseara y con la ayuda de Wagner iban desentrañando los desplazamientos y sustituciones que cada mito producía hasta cerrarse sobre sí mismo. A Roy le gustaba contar la versión más simplificada que encontraba en el cuento japonés del cantero que desea la suerte del rico y que en una serie de transformaciones concatenadas a partir de sus deseos de dominación vuelve, después de haber pasado por las posiciones de emperador, sol, nube, montaña y roca, a la posición de cantero, que moldea la roca a su antojo. Otro cuento popular más próximo, que permite una sencilla apropiación del procedimiento de obviación, es el de Cenicienta, quien tras una serie de sustituciones y desplazamientos situacionales (la madrina por la madrastra, la carroza por la calabaza, caballos por ratones, harapos por vestido de fiesta; de las cenizas al salón de baile, del zapato de cristal al resbalón en la escalera, del no queda tiempo a todo el tiempo del mundo...) termina por transformarse en lo que de algún modo ya era: una princesa, un ser amado-amante, ya no por su padre noble sino por su noble esposo. Estas pruebas ensanchan los límites de lo posible y re-definen lo convencional y a su vez lo singularizante. Wagner ofrece una versión invertida del cuento de Cenicienta, Splinterela (algo así como mujer entablillada), en Coyote Anthropology (2010: 124-125).

Coyote Anthropology, el más convencionalmente experimental libro del autor, nos enfrenta a un diálogo sobre la creación de sentido al más puro estilo de los metálogos de Bateson. En él Roy conversa con el Coyote -su doble invertido o anti-twin- sobre algunos de los conceptos que Wagner produce en diálogo con la obra de Castaneda. En el anti-twin o doble invertido fundamenta Wagner la fundación cultural del animal humano. Si las polaridades elementales, diferencia hacia adentro o lateralidad (derecha-izquierda) y diferencia hacia afuera o género (masculino-femenino) se invierten, es decir, si volteamos hacia dentro el género y volteamos hacia fuera la lateralidad, los efectos resultantes son respectivamente el incesto y la tecnología, los dos rasgos consustanciales al ser humano. El anti-twin, que estaría presente en nuestra base filogenética, en la doble hélice de nuestro ADN, sería la herencia quiasmática de la humanidad, resultado y a la vez principio de esas inversiones y desplazamientos mediante los cuales opera el conocimiento. La idea del anti-twin, tal y como podemos leer en otra de sus obras, An Anthropology of The Subject (2001: 53-54), la toma Wagner de Bateson, concretamente de una imagen del capítulo 3 "Múltiples versiones del mundo" de Espíritu y Naturaleza (Bateson 1993), en el que este último equipara la acción de juntar las palmas de las manos con el efecto de mirarse al espejo para diferenciar entre descripción, tautología y explicación y afirmar la importancia de los desplazamientos abductivos que permiten las tautologías. Estos desplazamientos, que eluden -podríamos decir, obvian- las relaciones que preceden a los fenómenos y operan solo en el nivel propositivo, estarían para Bateson en la base del conocimiento. La propuesta obviadora de la invención u obviación wagneriana converge en algún sentido por tanto con la ciencia del espíritu batesoniana, aunque sin duda toma caminos diferentes para su expresión.

En un texto que presenta la influencia de Bateson en Wagner, Elizabeth Stassinos (2002) considera que, a diferencia de Bateson, quien utiliza la biología como contrapunto para sus revelaciones etnográficas, Wagner se sirve de Castaneda y la ciencia ficción como heurísticos para sortear «las taras conceptuales de una antropología demasiado reacia a usar la teoría contra las perspectivas ofuscantes de su propia cultura» (ibid.: 67). A pesar de estas afecciones, es curioso constatar que no hay en los escritos de Wagner rasgo alguno del misticismo que encontramos en antropólogos que utilizan las ciencias como esos elementos exógenos de contraste, como es el caso de Bateson o el propio Lévi-Strauss. Recordemos cómo acaba Bateson el capítulo de Espíritu y Naturaleza referido arriba: «¿Cuál es mi respuesta a la pregunta acerca de la 
naturaleza del conocimiento? Me entrego a la creencia de que mi conocimiento es una pequeña parte de un conocimiento integral más amplio que entrama a toda la biosfera o creación» (Bateson 1993: 102). En el mismo sentido consideraba Lévi-Strauss que la estructura debía de ser un designio que excedía los contornos de la mente humana. La unidad sagrada postulada a través del contraste científico. La invención científica desentrañada a través de la ficción etnográfica. Si la obviación no deviene el concepto más apropiado para pensar de y desde la antropología es por razón de su propia operatividad.

Durante 30 años Roy Wagner ofreció una asignatura en UVa dedicada al trabajo de Carlos Castaneda: «Don Juan \& Castaneda», a la que asistí en 2016. El curso, dedicado a las descripciones alternativas de la realidad, tomaba como punto de partida los nueve primeros libros de Carlos Castaneda, desde Las enseñanzas de Don Juan, de 1968, hasta El arte de ensoñar, de 1993; y abordaba cuestiones como el poder de los cambios de perspectiva -la base del conocimiento antropológico-, la expersonation o acción de expandirse más allá del cuerpo; el humor como medio para ver; el doble como realidad intrínseca al enmascaramiento humano -una propuesta que Alberto Corsín Jiménez (2019) utiliza hábilmente en su reciente "Antropologías de tela de araña»-; la burbuja de la percepción; el concepto tolteco de Tonal y su negativo, el nagual; la comparación de doble proporcionalidad; el punto de ensamblaje (assemblage-point); la objetivación de la holografía; y una veintena de propuestas más que hacen de esta asociación entre Wagner y Castaneda una de las mayores vetas para la extracción de ingenio antropológico.

A pesar de que nunca se conocieron personalmente, Wagner estaba convencido de que El poder del silencio, el libro que Castaneda publicó en 1987, versaba sobre la obviación. El libro, dedicado al modo en que los naguales adquieren poder a través de las historias que cuentan, aborda el núcleo abstracto, el conocimiento silencioso de las historias. Para Wagner, la explicación que hace Don Juan de la fuerza de las historias como propósito o intención (intent) es virtualmente idéntica a la técnica de la obviación y estaba convencido de que Castaneda había replicado su idea, habiendo llegado a ella a través de su supervisor en la Universidad de California (UCLA), Phillip Newman, experto en el análisis de los mitos de Nueva Guinea. Una curiosa anécdota parece apoyar la tesis de Wagner. En el verano de 1993, una librera de Char- lottesville, Kay Allison, que regentaba la mí(s)tica Quest Bookshop, telefoneó a Roy para que acudiera inmediatamente a la librería. Al llegar, Wagner se encontró con que alguien había remitido allí cientos de ejemplares del libro de Castaneda, algo que para Wagner confirmaba que Castaneda había hecho uso no explícito de su argumentación y pagaba así por ello.

Además de una inteligencia privilegiada, Roy tenía una memoria prodigiosa y recordaba los nombres y aportaciones de todas y todos sus alumnos aventajados, además de cientos de anécdotas y precisos detalles de lo que había vivido a lo largo de su vida. Durante el semestre que pasé en Virginia, le acompañé en sus paseos por el campus, desde Brooks Hall, el edificio más peculiar de la universidad de Virginia y donde se encuentra el Departamento de Antropología, hasta Gibson Hall, donde daba la clase. Hablábamos en ellos del Beethoven célibe cargado de plomo, del Rilke heredero, del ergativo vasco, de la prueba ontológica de Gödel, de las novelas de Ursula K. LeGuin, de la elegancia de Pitt-Rivers, de las prácticas caníbales de los vecinos de los daribis o de cualquier otra cosa que sirviera para saciar la incontinencia verbal de Roy, que él mismo atribuía a su herencia paterna o incluso a su origen natal. Al parecer, Cleveland es un lugar en el que hablar se convierte en una práctica agónica. Pararse a saludar puede alargarse durante horas de apasionada lucha por las palabras. Hijo de un jefe de policía y a quien su madre desde pequeño le animaba a leer libros de los Kroeber, acabó siendo hanarubo para los daribis, «el que siempre está haciendo preguntas».

Roy Wagner, al igual que Bateson o Castaneda, no ha dejado discípulos directos, pero son ya legión quienes lo reivindican como uno de los antropólogos más originales del siglo XX. Pronto verá la luz el primer estudio de su obra, de la brasileña Iracema Dulley (2019). Y en lo que nos concierne por cercanía, se ha publicado recientemente The culture of invention in the Americas, un volumen editado por Pedro Pitarch y José Antonio Kelly (2018), que recoge las contribuciones a un seminario en honor a Wagner que se celebró en Trujillo en 2013. En castellano, Montserrat Cañedo (2013) incluyó el artículo «La persona fractal» en su Cosmopolíticas, y podremos leer, por fin, y nos ha costado casi medio siglo, La invención de la cultura.

Marilyn Strathern, a quien Wagner admiraba profundamente, pensó al leer sus textos que aquello solo podía haber sido escrito por alguien oscuro. «Yo no 
soy en absoluto oscuro», protestó Roy al contarme la anécdota. Ciertamente Roy no era en absoluto oscuro, más bien al contrario, era una persona luminosa. En los comentarios a su último artículo «La reciprocidad de perspectivas» (2018), Martin Holbraad apunta también a la luminosidad (en este caso de los textos) de Wagner, que nada tiene que ver para él con la claridad. Holbraad lo compara con un profeta quien «habla no del futuro sino desde él, así que hasta que ese tiempo llega permanece opaco, y cuando llega se pasa de moda» (2018: 514). A pesar de que Holbraad matiza esta última parte para afirmar que Wagner es inmune al paso del tiempo por efecto de su yo anticipatorio (anticipatory self), que proyecta sin predecir aquello que los lectores podrían querer encontrar en él, creemos que hay una imagen mejor que la del profeta para pensar la figura de Wagner. Recuperando la intuición de Strathern, podemos pensar en Roy Wagner como el espejo humeante de la antropología:

Una superficie de doble reflejo que incluye una capa de vidrio de obsidiana pulido, los Toltecas lo entendieron como una especie de puerta que conecta el mundo de los seres de energía (las «conciencias inorgánicas» de Castaneda) con el nuestro, $y$, en efecto, se articula en anti-duplicidad (antitwinning) como una agencia que puede actuar libremente, por su propia cuenta. Es como si el ser de energía o "aliado», que obtiene todo su sentido de ser desde su reflejo en el nuestro, se activara en el mundo en forma de los cuatro Hermanos Tezcatlipoca, manifestando las cuatro direcciones cardinales, como si el ego reflejado en el espejo de Lacan fuera elevado a un estado divino (Wagner 2019: 4).

Summa Antropologica. Para muchos, la opacidad del espejo humeante será impenetrable. Para otras y otros, una poderosa arma de conocimiento que vivirá gracias a ellos.

\section{BIBLIOGRAFÍA DE ROY WAGNER ${ }^{2}$}

1967. The Curse of Souw: Principles of Daribi Clan Definition and Alliance. Chicago: Chicago University Press.

1969. "Marriage among the Daribi», en Robert M. Glasse y Mervyn J. Meggitt, Pigs, Pearlshells, and Women: Marriage in the New Guinea Highlands: 56-76. Englewood Cliffs: Prentice Hall.

2 Esta bibliografía pretende ser exhaustiva. Aun así, teniendo en cuenta la abundante obra del autor, es probable que haya omitido involuntariamente alguna de sus contribuciones.
1971. (con Stanley Walens). «Pigs, Proteins, and People-Eaters». American Anthropologist 73(1): 269-270.

1972. Habu: The Innovation of Meaning in Daribi Religion. Chicago: University of Chicago.

1974. "Are There Social Groups in the New Guinea Highlands?», en Murray J. Leaf (ed.), Frontiers of Anthropology: 95-122. Nueva York: Van Nostraad.

1981 [1975]. The Invention of Culture. Chicago: University of Chicago Press.

1977. «Analogic Kinship: A Daribi Example». American Ethnologist 4(4): 623-642.

1977. «Scientific and Indigenous Papuan Conceptualizations of the Innate: A Semiotic Critique of the Ecological Perspective», en Timothy P. Bayliss-Smith y Richard G. Feachem (ed.), Subsistence and Survival: Rural Ecology in the Pacific: 385410. Londres/Nueva York: Academic Press.

1978. Lethal Speech: Daribi Myth as Symbolic Obviation. Ithaca: Cornell University Press.

1979. "The Talk of Koriki: a Daribi Contact Cult». Social Research 46: 140-165.

1980. «Ethnology: Do Kamo: Person and Myth in the Melanesian World. Maurice Leenhardt». American Anthropologist 82(3): 690-691.

1980. "Cultural Artifacts at Ornara and Kistobu Caves». Oral History 7(8): 59-63.

1982. "The Ri-Unidentified Aquatic Animals of New Ireland, Papua New Guinea». Cryptozoology 1(1): 33-39.

1984. "Ritual as Communication: Order, Meaning, and Secrecy in Melanesian Initiation Rites». Annual Review of Anthropology 13: 143-155.

1986. "The Theater of Fact and Its Critics». Anthropological Quarterly 59(2): 97-99.

1986. Symbols that stand for themselves. Chicago: University of Chicago Press.

1986. Asiwinarong: Ethos, Image, and Social Power among the Usen Barok of New Ireland. Princeton, NJ: Princeton University Press.

1987. «Figure-Ground Reversal among the Barok», en L. Lincoln (ed.), Assemblage of spirits: idea and image in New Ireland: 56-62. Minneapolis, MN: Minneapolis Institute of Arts.

1987. "Totemism», en Mircea Eliade (ed.), The Encyclopedia of Religion, vol. 15: 573-576. Nueva York: Macmillan.

1987. «Decoding Melanesia». Reviews in Anthropology 14(3): 215-220.

1988. "Visible Sociality: The Daribi Community», en James F. Weiner (ed.), Mountain Papuans: Historical and Comparative Perspectives from New Guinea Fringe Highlands Societies: 39-72. Ann Arbor, MI: University of Michigan Press.

1988. "Afterword», en Jadran Mimica, Intimations of Infinity: The Mythopoeia of the Iqwaye Counting System and Number: 162-163. Oxford: Berg. 
1989 (editado junto a Fred Damon). Death Rituals and Life in the Societies of the Kula. Dekalb: Northern Illinois University Press.

1991. "The Fractal Person», en Maurice Godelier and Marilyn Strathern (eds.), Big Men and Great Men: 159-173. Cambridge: Cambridge University Press

1991. "Poetics and the Recentering of Anthropology», en Ivan Brady (ed.), Anthropological Poetics: 37-50. Savage, MD: Rowman and Littlefield.

1991. «Dif/Ference and Its Disguises», en Mark C. Taylor, Robert P. Scharlemann, Roy Wagner, Michael Brint y Richard Rorty, On the Other: Dialogue and/or Dialectics: Mark Taylor's Paralectics: 42-52. Lanham: University Press of America.

1992. "The Imagery Keeps Its Scale: An Obviation Model of the Yafar Yangis», en Bernard Jullierat, Shooting the Sun: Ritual and Meaning in West Sepik: 206-213. Washington: Smithsonian Series in Ethnographic Inquiry.

1995. «Fighting over Pigshit: A New Ireland Pragmatic». Anthropology and Humanism 20(1): 3-8.

1995. "Hazarding Intent: Why Sogo Left Hweabi», en Lawrence Rosen (ed.), Other Intentions: Cultural Contexts and the Attribution of Inner States: 163-176. Santa Fe: School of American Research Press.

1995. "If You Have the Advertisement You Don't Need the Product», en Debbora Battaglia, Rhetorics of Self-Making: 59-76. Berkeley: University of California Press.

1995. «Fighting over pigshit: a New Ireland Pragmatic». Anthropology and Humanism 20(1): 3-8.

1995. "The Idea of Culture», en Walter Truett Anderson, The Truth about the Truth: Deconfusing and Reconstructing the Postmodern World: 53-57. Nueva York: Tarcher/Putnam.

1996. "Mysteries of Origin: Early Traders and Heroes in the Trans Fly», en Pamela Swadling, Plumes from Paradise: Trade Cycles in Outer Southeast Asia and Their Impact on New Guinea and Nearby Islands Until 1920: 285-298. Papua Nueva Guinea: Papua New Guinea National Museum in association with Robert Brown \& Associates.

2000. "The Reprojective Basis of Human Society». Soumen Antropologi. Journal of the Finish Anthropological Society 25(1): 4-14.

2000. «Our Very Own Cargo Cult». Oceania 70(4): 362-372.

2001. «Edith Turner: The Gender of Giftedness». Anthropology and Humanism 26(2): 190-194.

2001. An Anthropology of the Subject: Holographic Worldview in New Guinea and Its Meaning and Significance for the World of Anthropology. Berkeley: University of California Press.

2008. "Lost Horizons at Karimui», en Clifford Sather y Timo Kaartinen (eds.), Beyond the Horizon: Essays on Myth, History, Travel and Society: 75-88. Helsinki: Finnish Literary Society.
2009. "The Theory of Symbolic Obviation», en Jeppe Sinding Jensen (ed.), Myths and Mythologies. A reader: 308-320. Londres/Nueva York: Routledge Taylor and Francis Group.

2010. Coyote Anthropology. Lincoln: University of Nebraska Press.

2011. "The Chess of Kinship and the Kinship of Chess». HAU: Journal of Ethnographic Theory 1(1): 159-164.

2012. "Facts for You to Believe in Them; Perspectives Encourage You to Believe Out of Them. An Introduction to Viveiros de Castro's Magisterial Essay». HAU: Masterclass Series 1: 1-10.

2012. "Luck in the Double Focus: Ritualized Hospitality in Melanesia». Journal of the Royal Anthropological Institute 18: 161-174.

2012. "Figure-Ground Reversal among the Barok». HAU: Journal of Ethnographic Theory 2(1): 535-542.

2012. «Afterword: The Lottery of Babylon, or, the Logic of Happenstance in Melanesia and Beyond». Social Analysis 56(1): 165-175.

2013. "La Persona Fractal», en Montserrat Cañedo (ed.), Cosmopolíticas: Perspectivas Antropológicas: 83-98. Madrid: Trotta.

2014. "The Rising Ground». HAU: Journal of Ethnographic Theory 4(2): 297-300.

2018. "The Reciprocity of Perspectives». Social Anthropology 26(4): 502-510.

2019. The Logic of Invention. Chicago: HAU Books/University of Chicago Press.

\section{BIBLIOGRAFÍA CITADA}

Bateson, Gregory. 1993. Espíritu y Naturaleza. Buenos Aires: Amorrortu.

Cañedo, Montserrat. 2013. Cosmopolíticas. Madrid: Trotta.

Corsín Jiménez, Alberto. 2018. "Spider web Anthropologies: Ecologies, Infrastructures, Entanglements», en Marisol de la Cadena y Mario Blaser, A World of Many Worlds: 53-82. Durham: Duke University Press.

Dulley, Iracema. 2015. Os nomes dos outros: etnografia e diferença em Roy Wagner. Sao Paulo: Humanitas

Dulley, Iracema. 2019. On the Emic Gesture: Difference and Ethnography in Roy Wagner. Abingdon: Routledge.

Erikson, Philippe, Philippe Descola, Fernando Santos Granero, Laura Rival, Stephen Hugh-Jones, Carlos Fausto, Manuela Carneiro da Cunha, Harald Prins, Jonathan Hill, Anne Christine Taylor y Steve Rubenstein. 2008. "Discussion. Hommage á Claude Lévi-Strauss». Journal de la Société des Américanistes 94(2): 45-54.

González-Abrisketa, Olatz y Susana Carro-Ripalda. 2016. «La apertura ontológica de la antropología contemporánea». 
Revista de Dialectología y Tradiciones Populares 71(1): 101128. doi: <https://doi.org/10.3989/rdtp.2016.01.003>

Holbraad, Martin. 2018. "The Difficulty with Wagner». Social Anthropology 26(4): 513-514.

Holbraad, Martin y Morten Axel Pedersen. 2017. The Ontological Turn: An Anthropological Exposition. Cambridge: Cambridge University Press.

Ludvigson, Tomas. 1974. «Reseña de Habu: The Innovation of Meaning in Daribi Religion by Roy Wagner». Journal of the Polynesian Society 83(1): 117-119.

Pitarch, Pedro y José Antonio Kelly. 2018. The Culture of Invention in the Americas. Canon Pyon: Sean Kingston Publishing.

Stassinos, Elizabeth. 2002. "The Art of Losing One's Own Culture Isn't Hard to Master, It's Obviation: Roy Wagner, Gregory Bateson, and the Art of Science Writ Large». Social Analysis: The International Journal of Social and Cultural Practice 46(1): 62-68.

Wagner, Roy. 1968. The Curse of Souw: Principles of Daribi Clan Definition and Alliance in New Guinea. Chicago: University of Chicago Press.

Wagner, Roy. 1972. Habu: The Innovation of Meaning in Daribi Religion. Chicago: University of Chicago Press.
Wagner, Roy. 1978. Lethal Speech: Daribi Myth as Symbolic Obviation. Ithaca, NY/Londres: Cornell University Press.

Wagner, Roy. 1981. The Invention of Culture. Chicago: University of Chicago Press.

Wagner, Roy. 1986. Symbols That Stand for Themselves. Chicago/Londres: University of Chicago Press.

Wagner, Roy. 2001. An Anthropology of the Subject: Holographic Worldview in New Guinea and Its Meaning and Significance for the World of Anthropology. Berkeley: University of California Press.

Wagner, Roy. 2013. «La Persona Fractal», en Montserrat Cañedo (ed.), Cosmopolíticas: Perspectivas Antropológicas: 8398. Madrid: Trotta.

Wagner, Roy. 2019. The Logic of Invention. Chicago: HAU Books/ University of Chicago Press.

Weiner, James F. 1995. The Lost Drum: The Myth of Sexuality in Papua New Guinea and Beyond. Madison: University of Wisconsin Press.

Young, Michael. 1974. "Reseña de Habu: The Innovation of Meaning in Daribi Religion by Roy Wagner». Bulletin of the School of Oriental and African Studies 37(1): 266-268. 